ORIGINAL ARTICLE

\title{
Retrospective Treatment Outcomes of Concurrent Chemoradiotherapy in Patients with Stage III Non-small Cell Lung Cancer at a Single Institution: The Pre-PACIFIC Era
}

\author{
Mitsue Kawamural; Norio Araki ${ }^{1}$; Noriko Kishil; Tatsuya Suwal; Yuki Yamamoto2; \\ Osamu Kanai2; Kohei Fujita2; Misato Okamura2; Koichi Nakatani2; Tadashi Mio2
}

\begin{abstract}
Objective. To investigate the treatment outcomes in patients with stage III non-small cell lung cancer (NSCLC) after definitive concurrent chemoradiotherapy (CCRT). Methods. We retrospectively reviewed 89 patients who received definitive CCRT for NSCLC in our hospital between April 2008 and March 2018. According to the Union for International Cancer Control system, the clinical stages were IIIA in version 7, IIIB, IIIA in version 8 , IIIB, and IIIC in 44, 45, 29, 46, and 14 patients, respectively. Results. The 5-year overall survival (OS) and progression-free survival rates were $48.5 \%$ and $36.1 \%$, respectively. Five-year OS by stage was $57.0 \%$ for stage IIIA in version $7,41.7 \%$ for stage
\end{abstract}

IIIB ( $\mathrm{p}=0.14), 63.8 \%$ for stage IIIA in version $8,45.7 \%$ for stage IIIB, and $28.6 \%$ for stage IIIC $(\mathrm{p}=0.02)$. Conclusion. CCRT was feasible, safe, and well tolerated in our patients and resulted in survival benefits comparable to those in the published literature. Stage IIIC NSCLC (version 8) was associated with a significantly worse prognosis. Some stage IIIC cases suggested that radiation therapy should not be omitted, even for stage IIIC, and that it is better to increase the intensity of systemic therapy, including durvalumab.

(JJLC. 2021;61:383-388)

KEY WORDS — Lung cancer, CCRT, 3D-CRT

\section{INTRODUCTION}

Concurrent chemoradiotherapy (CCRT) has been the standard treatment for patients with locally advanced non-small cell lung cancer (NSCLC) ${ }^{1}$; however, the prognosis of these patients has been poor for two decades, with an estimated 5-year overall survival (OS) rate of $<20 \%$ (range, 15-40\%).2 The treatment strategy for stage III NSCLC is complex and controversial because of the heterogeneity of these patients. ${ }^{3-5}$ After the PACIFIC trial, durvalumab, which is a consolidation therapy for patients with stage III NSCLC with no progression after chemoradiotherapy, became the standard therapy. ${ }^{6,7}$

We herein present our single-institution study on the treatment of patients with NSCLC using CCRT: the prePACIFIC era. This retrospective study aimed to assess the outcomes of consecutive patients with locally advanced NSCLC, who were treated by only one boardcertificated radiation oncologist for ten years at our institution.

The outcomes in each subgroup were also assessed. The subgroups were categorised according to the malignant tumour classification of the 8th edition of the Union for International Cancer Control (UICC), which was changed in 2017.8,9 Determination of the therapeutic approach for stage III NSCLC was mainly based on the TNM classification system, and these outcomes will indicate the appropriate strategies for each subgroup.
${ }^{1}$ Department of Radiation Oncology, ${ }^{2}$ Department of Respiratory Medicine, National Hospital Organization Kyoto Medical Center, Japan.
Corresponding author: Norio Araki.

Received October 8, 2020; accepted June 7, 2021. 


\section{MATERIALS AND METHODS}

\section{Patient population}

Patients who were treated with definitive chemoradiotherapy for locally advanced NSCLC were eligible for inclusion in this retrospective review. The exclusion criteria were as follows: treatment with radiotherapy (RT) alone, treatment with surgery after chemoradiotherapy, history of other malignancy within 5 years, and absence of follow-up because of unavailability. The diagnosis of NSCLC was confirmed by histological evaluation. All patients were staged with whole-body fluorodeoxyglucosepositron emission tomography and enhanced magnetic resonance imaging of the brain. The clinical stage at presentation was subsequently categorised according to the TNM classification of malignant tumours (in both the 7 th and 8th editions).

Medical records and clinical laboratory data for the patients were retrospectively collected and anonymised to exclude personal information. The study protocol was approved by the Institutional Review Board of our hospital (19-030).

\section{Treatment and outcomes}

The patients received CCRT as initial treatment. The prescribed radiation dose was 60-66 Gy delivered in 2.0 Gy daily fractions, five times a week. Patients were treated with elective nodal irradiation therapy with three-dimensional conformal RT (3D-CRT), which prophylactically targeted the uninvolved mediastinal nodal regions. A shrinking field technique was used, with opposed anterior/posterior fields covering the primary tumour and elective mediastinal nodes, followed by the cone-down fields angle off the spinal cord. Patients were followed every 3 months for the first year and every 6 months thereafter. The toxicity of chemoradiotherapy was graded using the Common Terminology Criteria for Adverse Events (CTCAE) version 5.0.

\section{Statistical analysis}

OS was defined as the time from the date of first treatment to the date of death or date of the last contact. Progression-free survival (PFS) was defined as the time from the date of first treatment to the date of disease progression or death from any cause. Patient characteristics were retrospectively obtained from the medical records.

For the univariate analysis, OS was assessed using the Kaplan-Meier method and log-rank test. P-values of
Table 1. Patient Characteristics

\begin{tabular}{|c|c|c|}
\hline Age (years) & $46-79$ & (median 66) \\
\hline \multicolumn{3}{|l|}{ Sex } \\
\hline Male & 69 & $(77.5 \%)$ \\
\hline Female & 20 & $(22.5 \%)$ \\
\hline \multicolumn{3}{|l|}{ Histology } \\
\hline Adenocarcinoma & 37 & $(41.6 \%)$ \\
\hline Squamous cell carcinoma & 21 & $(23.6 \%)$ \\
\hline Others & 31 & $(34.8 \%)$ \\
\hline \multicolumn{3}{|l|}{ Stage (7th ed.) } \\
\hline IIIA & 44 & $(49.4 \%)$ \\
\hline IIIB & 45 & $(50.6 \%)$ \\
\hline \multicolumn{3}{|l|}{ Stage (8th ed.) } \\
\hline IIIA & 29 & $(32.6 \%)$ \\
\hline IIIB & 46 & $(51.7 \%)$ \\
\hline IIIC & 14 & $(15.7 \%)$ \\
\hline Total dose of radiotherapy (Gy) & $60-66$ & Median 60 \\
\hline \multicolumn{3}{|l|}{ Chemotherapy regimen } \\
\hline $\mathrm{CDDP} 60 \mathrm{mg} / \mathrm{m}^{2} / \mathrm{VNR} 25 \mathrm{mg} / \mathrm{m}^{2}$ & 47 & $(52.8 \%)$ \\
\hline CBDCA AUC $2 / \mathrm{PAC} 40 \mathrm{mg} / \mathrm{m}^{2}$ & 22 & $(24.7 \%)$ \\
\hline CBDCA AUC 6/nabPTX $100 \mathrm{mg} / \mathrm{m}^{2}$ & 9 & $(10.1 \%)$ \\
\hline Others & 11 & $(12.4 \%)$ \\
\hline
\end{tabular}

Abbreviations: CDDP, cisplatin; CBDCA, carboplatin; VNR, vinorelbine; PAC, paclitaxel; nabPTX, nab-paclitaxel.

$<0.05$ were considered statistically significant. Statistical analyses were performed using the EZR software program (version 1.31, Saitama Medical Center, Jichi Medical University, Saitama, Japan), which is a graphical user interface for $\mathrm{R}$ (the $\mathrm{R}$ Foundation for Statistical Computing, Vienna, Austria). ${ }^{10}$

\section{RESULTS}

We retrospectively identified 89 consecutive patients with locally advanced NSCLC, all of whom underwent definitive chemoradiotherapy in our hospital between April 2008 and March 2018. The characteristics of the patients are shown in Table 1. Activating EGFR mutations (exon 19 deletion and exon 21 L858R point mutation) were detected in 12 of 80 patients. These patients received two cycles of platinum-based chemotherapy concurrently with definitive RT. The dose of chemotherapy is shown in Table 1 . A total of 87 patients received consolidation chemotherapy with the same regimen.

The median follow-up period was 55 months (range, 3121 months) in all patients. A total of 56 patients had disease recurrence, and 47 patients died during follow-up. The median OS and PFS were 54.7 months (95\% confidence interval [CI], 36.4-79.5) and 22.7 months (95\% CI: 12.2-52.8), respectively. The 5-year OS and PFS were 

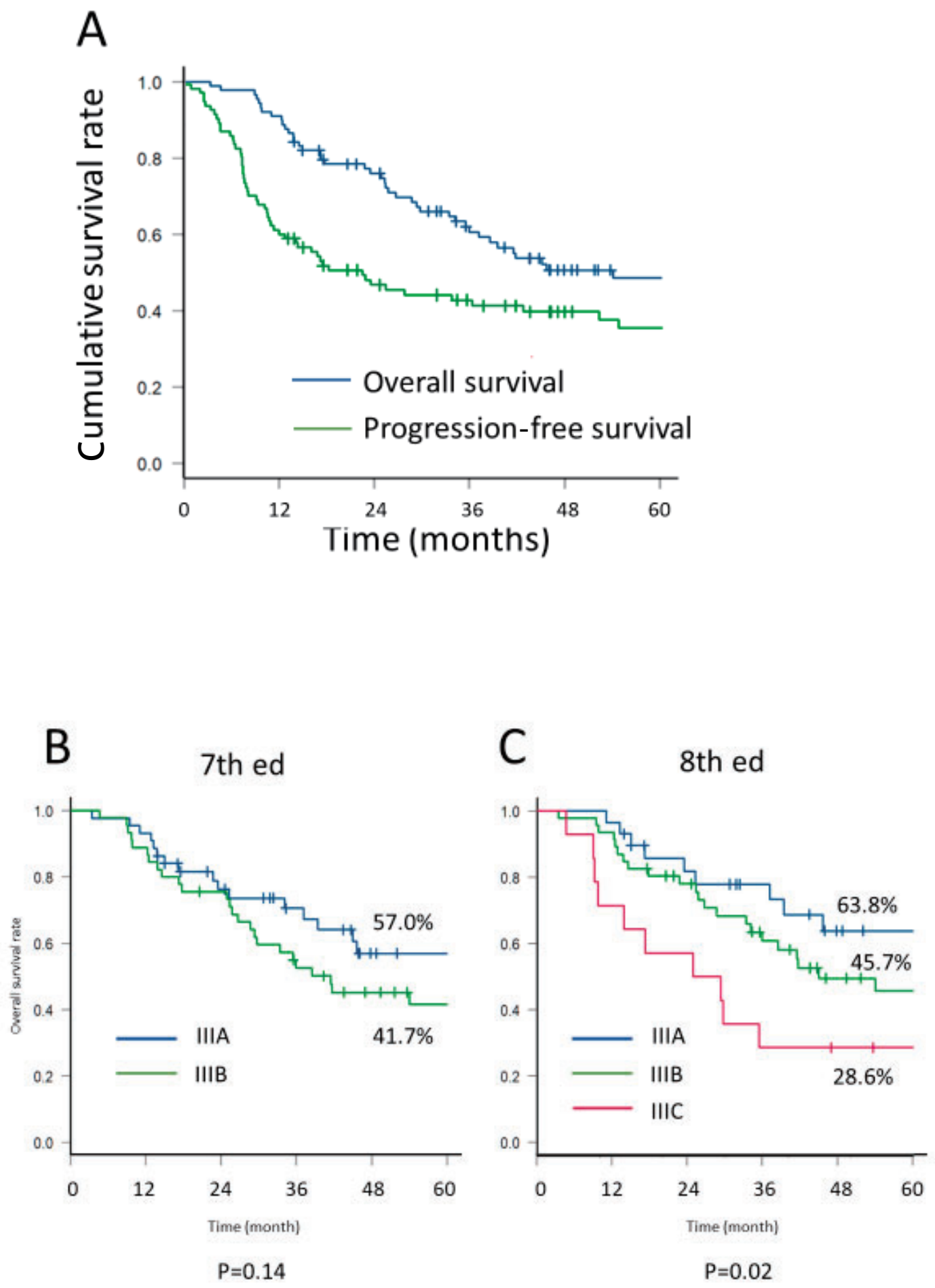

Figure 1. A. Kaplan-Meier curves of overall survival and progression-free survival in all patients. B, C. Difference in overall survival among the stages.

48.5\% and $36.1 \%$, respectively. The 5 -year OS by stage was $57.0 \%$ for stage IIIA (version 7 ), $41.7 \%$ for stage IIIB $(\mathrm{p}=0.14), 63.8 \%$ for stage IIIA (version 8 ), $45.7 \%$ for stage IIIB, and $28.6 \%$ for stage IIIC $(p=0.02)$ (Figure 1$)$. In the subgroup analysis, there was no significant difference in 5-year OS (Table 2). OS and PFS did not differ to a statistically significant extent according to the type of chemotherapy. Grade $>2$ radiation pneumonitis was observed 
Table 2. Subgroup Analysis

\begin{tabular}{lcccc}
\hline & Cut-off value & $\mathrm{N}(\%)$ & 5 -year OS (\%) & P-value \\
\hline Age & $<65$ years & & 62.7 & 0.06 \\
Sex & $>65$ years & $56(62.9 \%)$ & 41.0 & \\
& Male & & 49.4 & 0.91 \\
Histology & Female & $20(22.5 \%)$ & 45.7 & \\
& Adenocarcinoma & $37(41.6 \%)$ & 58.6 & 0.21 \\
T4 & Squamous cell carcinoma & $21(23.6 \%)$ & 37.5 & \\
& Others & $31(34.8 \%)$ & 44.9 & \\
N3 & $\leq 3$ & & 49.4 & 0.81 \\
& 4 & $28(31.5 \%)$ & 45.6 & \\
Radiation pneumonitis & $\leq 2$ & $35(39.3 \%)$ & 45.4 & 0.30 \\
& Grade $<1$ & & 50.5 & 0.31 \\
V20 Gy & Grade $\geq 2$ & $22(24.7 \%)$ & 43.8 & \\
& $<25 \%$ & & 49.0 & 0.95 \\
& $\geq 25 \%$ & $41(46.1 \%)$ & 48.4 & \\
\hline
\end{tabular}

Table 3. Radiation Pneumonitis

\begin{tabular}{lcccc}
\hline V20 Gy (\%) & N & G2 & G5 & $>$ G2 (\%) \\
\hline $0-15$ & 9 & 1 & 0 & 11.1 \\
$15-25$ & 40 & 9 & 0 & 22.5 \\
$25-35$ & 36 & 9 & 1 & 27.8 \\
$35<$ & 4 & 2 & 0 & 50.0 \\
\hline Total & 89 & 21 & 1 & 24.7 \\
\hline
\end{tabular}

in $24.7 \%$ of patients (Table 3 ). As other adverse effects of chemoradiotherapy, grade 3 and 4 bone marrow suppression was observed in 35 and 22 patients, respectively, and grade 2 esophagitis was observed in 33 patients.

\section{RECURRENCE PATTERN}

At the time of data cut-off (28 May 2019), PFS events were observed in 58 patients, and 43 patients died. Among 58 patients with recurrence, locoregional failure was observed in 28 patients. Distant metastasis was the first site of failure in 30 patients. As for stage IIIC, distant metastasis occurred at a higher rate of $72.7 \%$ (Table 4). Moreover, 44 patients received chemotherapy, 17 received palliative RT, and 16 received immunotherapy as salvage therapy (some cases overlapped).

\section{DISCUSSION}

The outcomes reported in this retrospective study suggest that CCRT is feasible, safe, and well tolerated. The results also demonstrate that CCRT was associated with a survival benefit in comparison to reports in the relevant literature. Stage IIIC (UICC 8 th edition) patients showed a significantly worse prognosis.

Stage III NSCLC includes a highly heterogeneous group of patients with differences in the extent and localisation of the disease. Many aspects of the treatment of stage III disease are controversial. These patients are at high risk for both local and distant recurrence. Even with major improvements in therapy, including the use of more active chemotherapy agents and refinements in radiation, the prognosis for these patients has remained poor for two decades. RTOG0617 revealed that the delivery of 74 Gy with concurrent carboplatin and paclitaxel resulted in worse median OS (20 months versus 29 months) in comparison to the delivery of 60 Gy. ${ }^{11,12}$ This study was a two-by-two phase 3 randomised trial to compare a standard dose of $60 \mathrm{~Gy}$ and a high dose of 74 Gy and concurrent chemotherapy with or without cetuximab in patients with inoperable stage III NSCLC. As a result, high-dose RT was associated with shorter survival and increased risk of death in comparison to conventional-dose RT. A multivariate analysis suggested that poorer survival may be related to a higher dose of radiation to the heart. However, higher doses of RT will be beneficial when chemotherapy cannot be indicated. Recently, a paradigm shift has occurred. The PACIFIC trial results showed that the administration of durvalumab, an anti-programmed death ligand 1 monoclonal antibody, after CRT in patients with unresectable stage III significantly prolonged PFS and OS in compari- 
Table 4. Recurrence Pattern

\begin{tabular}{lcccc}
\hline \multicolumn{1}{c}{ Recurrence pattern } & IIIA & \multicolumn{1}{c}{ IIIB } & IIIC & All patients \\
\hline Local recurrence & $6(37.5 \%)$ & $11(35.5 \%)$ & $2(18.2 \%)$ & $19(33.9 \%)$ \\
Regional lymph node & $4(25.0 \%)$ & $4(12.9 \%)$ & $1(9.1 \%)$ & $9(16.1 \%)$ \\
Metastasis & $6(37.5 \%)$ & $16(51.6 \%)$ & $8(72.7 \%)$ & $30(57.1 \%)$ \\
Lung & 4 & 6 & 2 & 12 \\
Brain & 1 & 4 & 4 & 9 \\
Bone & 1 & 4 & 0 & 5 \\
Liver & 0 & 1 & 2 & 3 \\
Adrenal gland & 0 & 1 & 0 & 1 \\
\hline Total & $16 / 29$ & $31 / 46$ & $11 / 14$ & $58 / 89$ \\
\hline
\end{tabular}

son to placebo. ${ }^{6,7}$ In the subgroup analysis, the contribution of durvalumab was higher in the stage IIIA (7th edition) than in stage IIIB ( 7 th edition), which included stage IIIC (8th edition). This therapy has become the standard therapy for all stage III NSCLC. Durvalumab also had a favourable effect on the frequency of new distant metastasis, and was associated with a lower incidence of new brain metastasis.

The UICC staging system changed in 2017. The new dataset of the UICC 8 th edition contains a higher proportion of clinical cases from Asia, mostly from Japan, which contributed to $41 \%$ of the total cases. ${ }^{13}$ A new stage group was created for the most advanced local disease categories, T3 and T4 associated with N3 disease, but category M0. Such cases are presently classified as stage IIIC, reflecting their worse outcomes in comparison to cases involving tumours that remain in stage IIIB. The prognosis for stage IIIC cases is similar to that of stage IVA cases, but the separation is justified by the different treatment approaches used in such cases. Due to the changes in T category, despite the association of N2 or N3 disease, the change in stage classification, which has been proposed in the 8th edition, resulted in the shift from IIIA to IIIB and from IIIB to IIIC. In our study, the classification of the 8 th edition was well reflected in the prognosis. As the feature of stage IIIC, a higher rate of metastatic recurrence leads to a worse prognosis. The post-treatment outcomes of some stage IIIC cases suggested that RT should not be omitted, even in stage IIIC; thus, this may be a better strategy to increase the intensity of systemic therapy, including durvalumab.

The present study was associated with some limitations. First, because this was a retrospective study in a single institution, an information bias was inevitable, and the sample size was small. Second, patients with recurrence had the benefit of immunotherapy after nivolumab was approved for clinical use in 2014. A larger, multicentre analysis is warranted to discuss the strategy for unresectable stage III NSCLC. One merit of this study was that the RT policy had not changed for ten years. Furthermore, at the time of data cut-off (28 May 2019), only three patients were lost to follow-up, and these data were considered to reflect the real world setting.

\section{CONCLUSION}

CCRT was feasible, safe, and well tolerated in our patients and resulted in survival benefits that were comparable to those reported in the relevant literature. Stage IIIC (UICC 8th edition) was associated with a significantly worse prognosis. A few stage IIIC cases suggested that radiation therapy should not be omitted, even in stage IIIC, and that it is a better strategy for increasing the intensity of systemic therapy, including durvalumab.

本論文内容に関連する著者の利益相反：なし

Acknowledgements: This work was supported by the Kyoto Radiation Oncology Study Group.

Ethical statement: This study was approved by the local ethics committee of the National Hospital Organization Kyoto Medical Center of 19-030.

Presentation: This study was presented at the 2019 annual meeting of the Japanese Society of Radiation Oncology, Nagoya, Japan. 


\section{REFERENCES}

1. McCloskey P, Balduyck B, Van Schil PE, Faivre-Finn C, O’Brien M. Radical treatment of non-small cell lung cancer during the last 5 years. Eur J Cancer. 2013;49:15551564.

2. Curran WJ Jr, Paulus R, Langer CJ, Komaki R, Lee JS, Hauser S, et al. Sequential vs. concurrent chemoradiation for stage III non-small cell lung cancer: randomized phase III trial RTOG 9410. J Natl Cancer Inst. 2011;103: 1452-1460.

3. Yoon SM, Shaikh T, Hallman M. Therapeutic management options for stage III non-small cell lung cancer. World J Clin Oncol. 2017;8:1-20.

4. García-Campelo R, Bernabé R, Cobo M, Corral J, Coves J, Dómine M, et al. SEOM clinical guidelines for the treatment of non-small cell lung cancer (NSCLC) 2015. Clin Transl Oncol. 2015;17:1020-1029.

5. Ozcelik M, Korkmaz T, Odabas H, Gemici C, Ercelep O, Yuksel S, et al. Comparison of efficacy and safety of three different chemotherapy regimens delivered with concomitant radiotherapy in inoperable stage III nonsmall cell lung cancer patients. Tumour Biol. 2016;37:89018907.

6. Antonia SJ, Villegas A, Daniel D, Vicente D, Murakami $\mathrm{S}$, Hui R, et al. Durvalumab after chemoradiotherapy in stage III non-small-cell lung cancer. N Engl J Med. 2017; 377:1919-1929.

7. Antonia SJ, Villegas A, Daniel D, Vicente D, Murakami S, Hui R, et al. Overall Survival with Durvalumab after Chemoradiotherapy in Stage III NSCLC. $N$ Engl J Med.
2018;379:2342-2350.

8. Sobin LH, Gospodarowicz MH, Wittekind C, eds. TNM Classification of malignant tumours (UICC International Union against Cancer). 7th ed. Oxford: Wiley-Blackwell; 2009.

9. Brierley JB, Gospodarowicz MH, Wittekind C, eds. TNM Classification of malignant tumours (UICC International Union against Cancer). 8th ed. Oxford: Wiley-Blackwell; 2017.

10. Kanda Y. Investigation of the freely available easy-to-use software 'EZR' for medical statistics. Bone Marrow Transplant. 2013;48:452-458.

11. Bradley JD, Paulus R, Komaki R, Masters G, Blumenschein G, Schild S, et al. Standard-dose versus high-dose conformal radiotherapy with concurrent and consolidation carboplatin plus paclitaxel with or without cetuximab for patients with stage IIIA or IIIB non-smallcell lung cancer (RTOG 0617): a randomised, two-by-two factorial phase 3 study. Lancet Oncol. 2015;16:187-199.

12. Movsas B, Hu C, Sloan J, Bradley J, Komaki R, Masters $\mathrm{G}$, et al. Quality of life analysis of a radiation doseescalation study of patients with non-small-cell lung cancer: a secondary analysis of the Radiation Therapy Oncology Group 0617 randomized clinical trial. JAMA Oncol. 2016;2:359-367.

13. Goldstraw P, Chansky K, Crowley J, Rami-Porta R, Asamura H, Eberhardt WE, et al. The IASLC Lung Cancer Staging Project: Proposals for Revision of the TNM stage groupings in the forthcoming (eighth) edition of the TNM classification for lung cancer. J Thorac Oncol. 2016;11:39-51. 